\title{
Editorial
}

\section{Optofluidics for Lab-on-a-Chip}

\author{
Eric P. Y. Chiou, ${ }^{1}$ Aaron T. Ohta, ${ }^{2}{\text { Zhihong } \text { Li, }^{3} \text { and Steven T. Wereley }}^{4}$ \\ ${ }^{1}$ Department of Mechanical and Aerospace Engineering, University of California, Los Angeles, CA, USA \\ ${ }^{2}$ Department of Electrical Engineering, University of Hawaii at Manoa, Honolulu, HI, USA \\ ${ }^{3}$ National Key Laboratory of Science and Technology on Micro/Nano Fabrication, Institute of Microelectronics, \\ Peking University, Beijing, China \\ ${ }^{4}$ Birck Nanotechnology Center, Purdue University, West Lafayette, IN, USA
}

Correspondence should be addressed to Eric P. Y. Chiou, pychiou@seas.ucla.edu

Received 22 December 2011; Accepted 22 December 2011

Copyright (C 2012 Eric P. Y. Chiou et al. This is an open access article distributed under the Creative Commons Attribution License, which permits unrestricted use, distribution, and reproduction in any medium, provided the original work is properly cited.

Optofluidic devices are gaining popularity due to the flexibility and ease of optical actuation and detection. These features are important for miniaturized lab-on-a-chip systems, which are often tricky to interface with larger benchtop equipment. Droplets, fluids, and particles can be manipulated using optofluidic devices. Simply adjusting the optical source can often reconfigures the type of manipulation, making optofluidics very adaptable compared to other methods. One unique feature of this special issue is its focus on indirect optical manipulation methods, in which light energy is used to trigger electric field, generate heat, or trigger rapidly expanding bubbles to achieve various manipulation functions, instead of using direct optical forces.

"Light-driven droplet manipulation technologies for labon-a-chip applications" by S.-Y. Park and E. P. Y. Chiou reviews various optical methods for droplet manipulation. Digital microfluidics is quite popular due to the compartmentalization and reduced cross-contamination provided by individual droplets. "Optoelectrofluidic manipulation of nanoparticles and biomolecules" by H. Hwang and J.-K. Park reviews techniques that combine optical and electrical effects in fluidic environments for the manipulation of nanoparticles and biomolecules such as proteins and DNA.

"Optoelectronic heating for fabricating microfluidic circuitry" by G. Haulot and C.-M. Ho demonstrates the formation of microfluidic channels by using optoelectronic heaters to melt frozen liquids. This can potentially be used to create fluidic structures on demand. "Moldless PEGDA-based optoelectrofluidic platform for microparticle selection" by S.-M. Yang et al. also presents a method for forming microfluidic channels; in this case, using UV-initiated polymerization for creating channels on an optoelectronic device designed to select individual microbeads.

Along with these papers of this issue, other papers involve dielectrophoresis and optically induced dielectrophoresis. The paper by P.-F. Wu and G.-B. Lee uses these two forces to arrange carbon nanotubes between electrodes, towards the goal of creating carbon nanotube sensors. The paper by P. J. Pauzauskie et al. also uses optically induced dielectrophoresis to trap nanotubes, which are detected in situ using Raman spectroscopy. The paper by K.-W. Huang et al. presents transparent electrodes that can be used to integrate devices for optically induced dielectrophoresis with microfluidic channels.

"An optically controlled $3 D$ cell culturing system" by K. $\mathrm{S}$. Ishii et al. uses optothermal heating to trigger a polymer phase transition that can be used to trap cells that are to be cultured. This can potentially be used to create cell cultures with minimal contamination. "From spheric to aspheric solid polymer lenses: a review" by K.-Y. Hung et al. presents methods for creating lenses for optical and optofluidic devices. The paper by D. McIntosh et al. demonstrates a microfluidic chip integrated with photodiode-based fluorescence detector that was used to detect the concentration of an antibiotic. 
Together, the papers in this special issue cover a wide range of topics in the growing field of optofluidics.

Eric P. Y. Chiou

Aaron T. Ohta

Zhihong $\mathrm{Li}$

Steven T. Wereley 

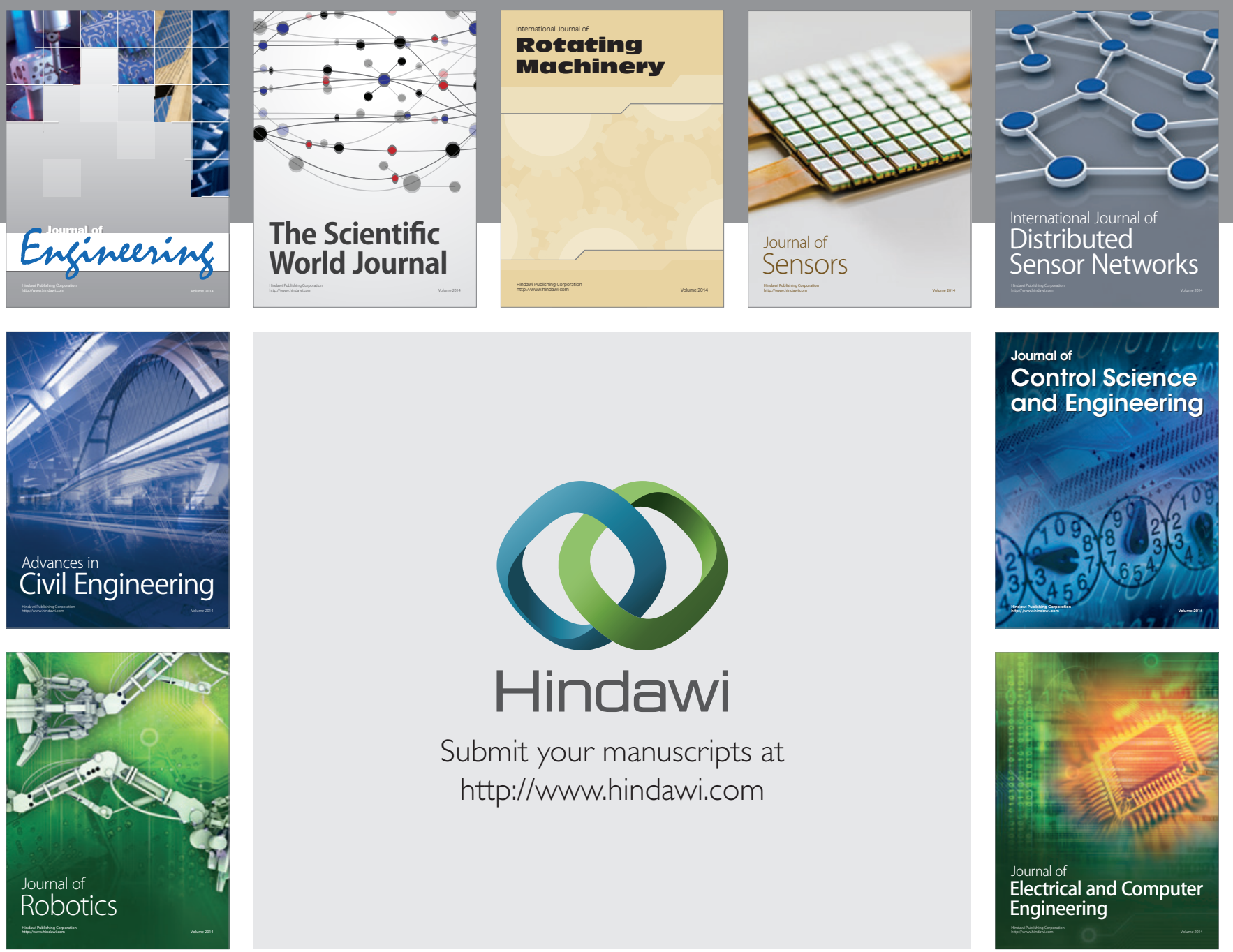

Submit your manuscripts at

http://www.hindawi.com
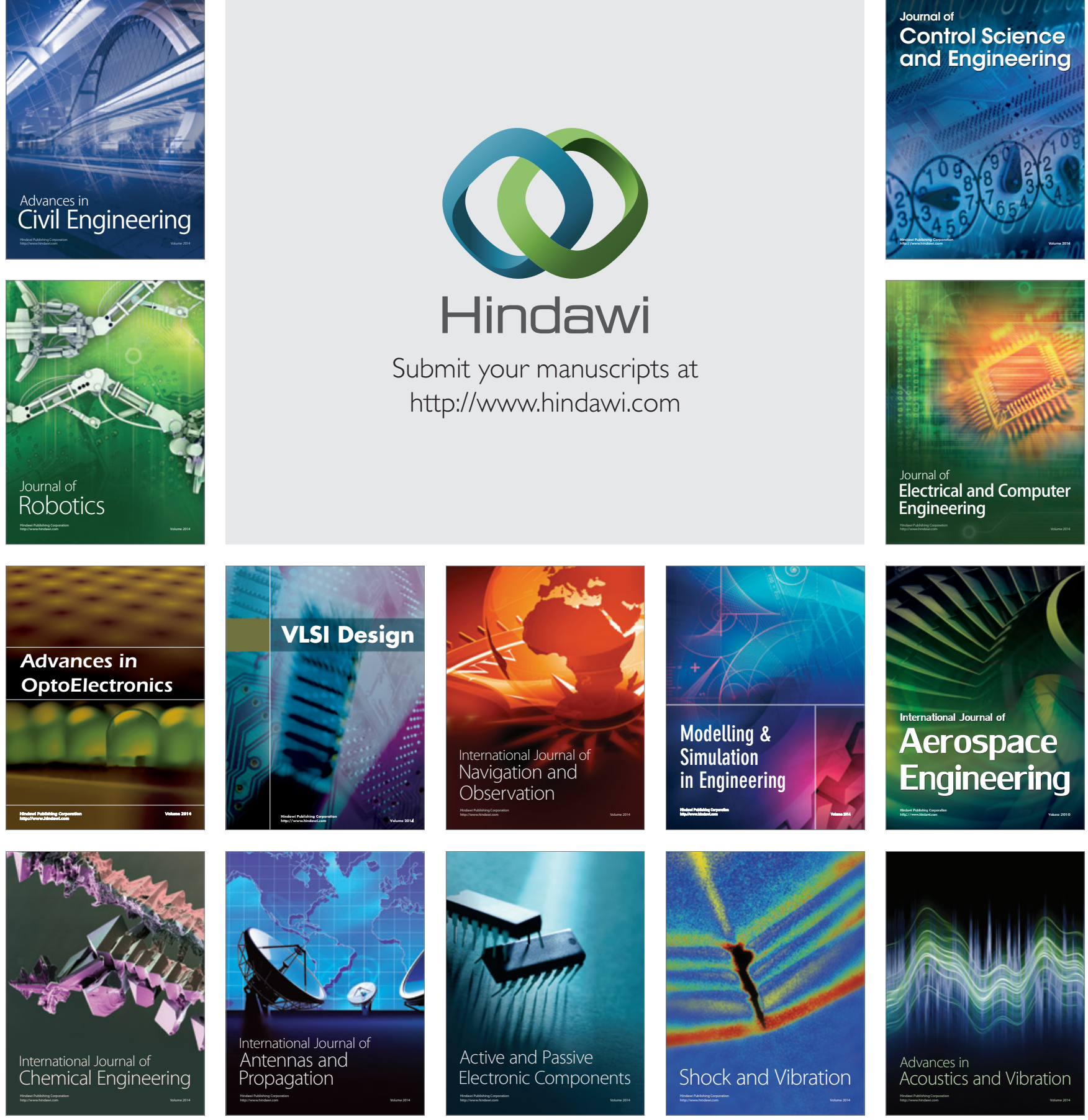